\title{
Interference Temperature Constraint-based Radio Resource Allocation for the Network Coded Cognitive Cooperative Network (NCCCN)
}

\author{
M. Shamim Kaiser \\ Institute of Information Technology \\ Jahangirnagar University \\ Dhaka-1342, Bangladesh
}

\author{
Shamim Al Mamun \\ Institute of Information Technology \\ Jahangirnagar University \\ Dhaka-1342, Bangladesh
}

\author{
Kazi M. Ahmed \\ Telecommunicatons, SET \\ Asian Institute of Technology \\ Pathumthani-12120, Thailand
}

\begin{abstract}
Demand for higher data rate wireless applications has led to scarcity in radio frequency spectrum. Spectrum access achieves near-optimal spectrum utilization efficiency with the advent of cognitive radio technology. In cognitive radio network (CRN), each cognitive user, also called secondary user (SU), senses and uses radio spectrum opportunistically while regulating the interference constraint. This article focuses the radio resource allocation considering interference temperature constraints for the network coded cognitive cooperative network (NCCCN). Analog network coded (ANC) Orthogonal-Frequency-DivisionMultiplexing (OFDM) improves the capacity of the cognitive cooperative network (CCN). Moreover, $\mathrm{CCN}$ enhances the spectrum utilization efficiency. Power allocation optimization problems have been formed that maximize the data transmission rate of NCCCN under the total transmit and peak-interference powers or the total transmit and average-interference powers. The spectral efficiency of the proposed network is compared with the spectral efficiency of CCN without ANC. Simulation results show that the proposed NCCCN enhances spectral efficiency in compared to the $\mathrm{CCN}$ without ANC.
\end{abstract}

\section{General Terms:}

Numerical Analysis, Radio Resource Allocation

\section{Keywords:}

Cognitive radio, power allocation, analog network coding, interference temperature, spectrum utilization

\section{INTRODUCTION}

Radio frequency spectrum is a natural resource for wireless networks. Government agencies allocate this licensed spectrum to wireless applications. This licensed band is divided into several sub-band and then allocated to wireless nodes, called primary users $(P U s)$, of that application. With the wide spread deployment of various wireless applications radio spectrum has become a scare resource. After scanning the radio frequency spectrum, it would be found that some sub-band are unoccupied or partially occupied[1, 13]. This leads to under utilization of scare radio spectrum [6 13]. Cognitive radio technology offers a novel technique to increase spectrum utilization efficiency [12, 9, 14]. In a cognitive radio network, secondary users $(S U s)$ are capable of sensing the radio environment to identify unoccupied or par- tially occupied sub-bands and then provides a mean for making those sub-bands available for $S U s$. The $S U s$ coexist with $P U s$ as long as the interferences to the $P U s$ are below specific thresholds [9, 14, 11].

The next Generation $(\mathrm{xG})$ wireless network demands high quality of service (QoS) in the multi-path fading environment [7]. Analog network coding (ANC) is a promising technique to improve capacity in wireless network. Orthogonal-Frequency-DivisionMultiplexing (OFDM) improves system performance by reducing inter-symbol interference of multi-path propagation environment. Thus ANC and ODFM are promising techniques to improve the QoS of the $\mathrm{xG}$ wireless network.

A single wireless link with multiple antenna improves spectral efficiency greatly. Multiple antenna concept may be impractical for portable mobile node, because of limitation on size and power. To overcome this limitation cooperative transmission has been proposed [9]. It utilizes the broadcast nature of the wireless communication channel and realize the almost similar performance of multiple antenna in each node.

ANC based protocol cooperative relaying is proposed for wireless sensor network in [2]. Authors showed that significant gain and throughput have been achieved with the use of network coding [15]. Outage probability analysis of cooperative relaying protocol with and without network coding has been studied in [3]. The power allocation optimization problem is formulated under transmit power and interference constraints for the underlay transmission [14]. They proposed an optimal power allocation (OPA) policy for multi-carrier cognitive radio network. In [9], authors have allocated power to OFDM based cognitive cooperative network $(\mathrm{CCN})$. They found that the spectral efficiency of the $\mathrm{CCN}$ is better than that proposed in [14].In [[4], [5]], Interference temperate constraints were modeled for spectrum access in CRNs. The authors proposed a non-binary receiver centric constraint model for spectrum access.

Power allocation considering ANC and interference constraint for OFDM based CCN has not been investigated. The power allocation in the underlay $\mathrm{CCN}$ is different from the conventional power allocation algorithms. In this paper, the capacity maximization problem subject to transmit and interference condition is also two fold like the optimization problem proposed in [9]. We demonstrate that our proposed approach improves spectrum utilization efficiency compared to traditional approaches.

The paper is organized as follows: the system model is presented in section II; power allocation algorithm is explained in section 


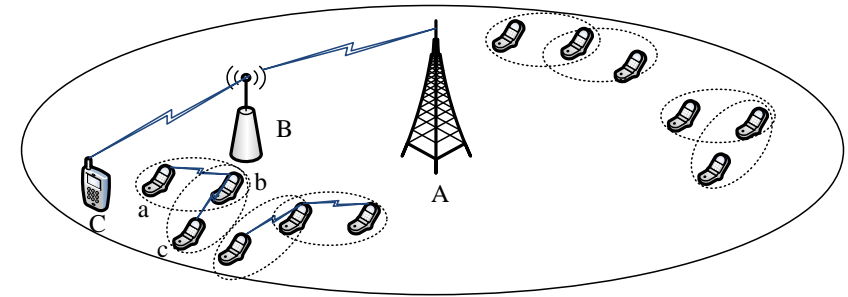

Fig. 1. A considered NCCCN where a primary user $A$ communicates with user $B$ via user/node $C$. Sharing the same channel, A secondary user $a$ communicates with user $b$ via user $c$.

III; Section IV presents discussion and results. The concluding remarks and future work is included in section $\mathrm{V}$.

\section{SYSTEM MODEL}

Fig. 1 shows a distributed deployment of a $\mathrm{CCN}$ which lies in the coverage area of a relay based cellular network. Cognitive radios or $S U s$ form ad-hoc network which share radio spectrum with $P U s$ in cooperative manner. In order to form power allocation optimization problem, we consider two $S U s$ (denoted by a and $b$ ) communicates with each other via a best positioned relay (denoted by $c$ ) whereas a mobile station (denoted by $A$ ) communicates with the base station (denoted by $B$ ) via a best positioned relay (denoted by $C$ ). In the first time slot, wireless node $\Im$ transmits and wireless nodes $\Re$ and $\aleph$ receive; in the second time shot, wireless node $\aleph$ transmits and wireless nodes $\Re$ and $\Im$ receive; in the third time slot $\Re$ forwards the transmission of $\Re$ and $\aleph$ (Fig.2). Here $\Im \in\{A, a\}, \aleph \in\{B, b\}$ and $\Re \in\{R, r\}$ The total number of OFDM subcarriers is $N$. It is assumed that the channel fading model is multipath Rayleigh, amplify-and-forward (AF) relaying is employed at the relay node, channel coefficients are symmetric, complex additive white gaussian noise with mean zero and variance $\sigma^{2}$ and each wireless node has learnt required parameter form common signaling control channel (CSCC). Using similar deduction of [8], the signal to noise plus interference (SNIR) of the received signal at the node $b$ and $a$ of the NCCCN, i.e., $\gamma_{a \rightarrow b}^{n}$ and $\gamma_{b \rightarrow a}^{n}$, in the three time slot is given by

$$
\begin{aligned}
\gamma_{a \rightarrow b}^{n}= & \frac{\kappa^{n} P_{c}^{n}\left|h_{a, b}^{n}\right|^{2}}{\sigma^{2}+\mu^{n} P_{p}^{n}\left|g_{A, b}^{n}\right|^{2}} \\
& +\frac{\frac{\kappa^{n} P_{c}^{n}\left|h_{a, c}^{n}\right|^{2}}{\sigma^{2}+\mu^{n} P_{p}^{n}\left|g_{A, c}^{n}\right|^{2}} \frac{\left(1-\kappa^{n}\right) P_{c}^{n}\left|h_{c, b}^{n}\right|^{2}}{\sigma^{2}+\left(1-\mu^{n}\right) P_{p}^{n}\left|g_{C, b}^{n}\right|^{2}}}{\frac{\kappa^{n} P_{c}^{n}\left|h_{a, c}^{n}\right|^{2}}{\sigma^{2}+\mu^{n} P_{p}^{n}\left|g_{A, c}^{n}\right|^{2}}+2 \frac{\left(1-\kappa^{n}\right) P_{c}^{n}\left|h_{c, b}^{n}\right|^{2}}{\sigma^{2}+\left(1-\mu^{n}\right) P_{p}^{n}\left|g_{C, b}^{n}\right|^{2}}}
\end{aligned}
$$

and

$$
\begin{aligned}
\gamma_{b \rightarrow a}^{n}= & \frac{\left(1-\kappa^{n}\right) P_{c}^{n}\left|h_{b, a}^{n}\right|^{2}}{\sigma^{2}+\left(1-\mu^{n}\right) P_{p}^{n}\left|g_{B, a}^{n}\right|^{2}} \\
& +\frac{\frac{\left(1-\kappa^{n}\right) P_{c}^{n}\left|h_{b, c}^{n}\right|^{2}}{\sigma^{2}+\left(1-\mu^{n}\right) P_{p}^{n}\left|g_{B, c}^{n}\right|^{2}} \frac{\kappa^{n} P_{c}^{n}\left|h_{c, a}^{n}\right|^{2}}{\sigma^{2}+\mu^{n} P_{p}^{n}\left|g_{C, a}^{n}\right|^{2}}}{\frac{\left(1-\kappa^{n}\right) P_{c}^{n}\left|h_{b, c}^{n}\right|^{2}}{\sigma^{2}+\left(1-\mu^{n}\right) P_{p}^{n}\left|g_{B, c}^{n}\right|^{2}}+2 \frac{\kappa^{n} P_{c}^{n}\left|h_{c, a}^{n}\right|^{2}}{\sigma^{2}+\mu^{n} P_{p}^{n}\left|g_{C, a}^{n}\right|^{2}}}
\end{aligned}
$$

where $\mu^{n}$ and $\kappa^{n}$ are power allocation proportional factors of the $P U$ and $S U$ respectively, $P_{p}^{n}$ and $P_{c}^{n}$ are the total link power of the $P U$ and $S U$ respectively, $h_{u, v}^{n}$ is the channel gain of the $u \rightarrow v$ link at $n$-th subcarrier, where $(u, v) \in$ $\{(a, c),(b, c),(a, b),(b, a),(c, a),(c, b)\}, g_{U, v}^{n}$ is the channel gain of the $U \rightarrow v$ interference link at $n$-th subcarrier, where $(U, v) \in\{(A, c),(B, c),(A, b),(B, a),(C, a),(C, b)\}$. The op-

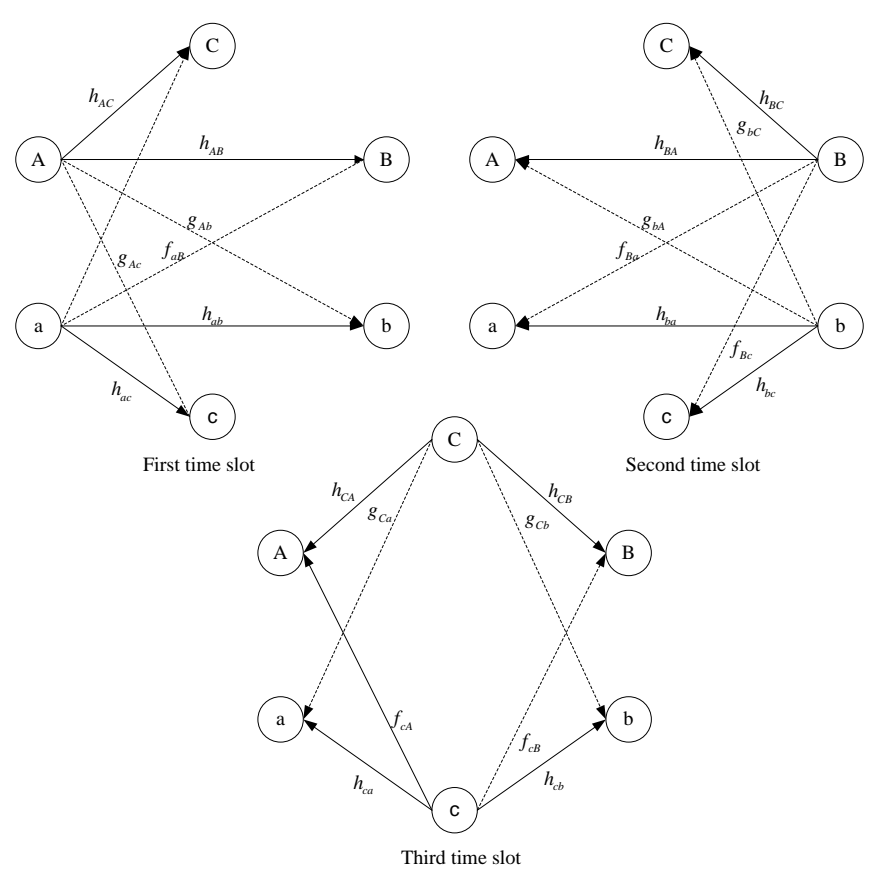

Fig. 2. A simplified version of Fig. 1 where left node transmits information to relay and right node in the 1st time slot. Right node transmits information to relay and left node in the 2nd time slot.

Relay node forwards the information in the 2 rd time slot.

timal value of $\mu$ and $\kappa^{n}$ can be determined using Eqn. (10) of [8].

Eqn. (1) can be simplified as

$$
\begin{aligned}
\gamma_{a \rightarrow b}^{n} & =\frac{\kappa^{n} P_{c}^{n}\left|h_{a, b}^{n}\right|^{2}}{\sigma^{2}+\mu^{n} P_{p}^{n}\left|g_{A, b}^{n}\right|^{2}} \\
& +\frac{P_{c}^{n} \frac{\left(1-\kappa^{n}\right)\left|h_{c, b}^{n}\right|^{2} \kappa^{n}\left|h_{a, c}^{n}\right|^{2}}{\left(1-\kappa^{n}\right)\left|h_{c, b}^{n}\right|^{2}+2 \kappa^{n}\left|h_{a, c}^{n}\right|^{2}}}{\sigma^{2}+P_{p}^{n}\left|g_{2}^{n}\right|^{2}}
\end{aligned}
$$

where $\left|g_{2}^{n}\right|^{2}=\frac{2\left(1-\mu^{n}\right)\left|g_{C, b}^{n}\right|^{2} \kappa^{n}\left|h_{a, c}^{n}\right|^{2}+\left(1-\kappa^{n}\right) P_{c}^{n}\left|h_{c, b}^{n}\right|^{2} \mu^{n}\left|g_{A, c}^{n}\right|^{2}}{\left(1-\kappa^{n}\right)\left|h_{c, b}^{n}\right|^{2}+2 \kappa^{n}\left|h_{a, c}^{n}\right|^{2}}$. In the worst case, the maximum equivalent channel gain of the interference link from $P U$ to $S U$, i.e., $\left|g^{n}\right|^{2}$, is given by $\left|g^{n}\right|^{2}=\max \left(\mu^{n}\left|g_{A, b}^{n}\right|^{2},\left|g_{2}^{n}\right|^{2}\right)$ and the equivalent channel gain of acb link, i.e., $\left|h^{n}\right|^{2}$, is given by $\left|h^{n}\right|^{2}=\kappa^{n}\left|h_{a, b}^{n}\right|^{2}+$ $\frac{\left(1-\kappa^{n}\right)\left|h_{c, b}^{n}\right|^{2} \kappa^{n}\left|h_{a, c}^{n}\right|^{2}}{\left(1-\kappa^{n}\right)\left|h_{c, b}^{n}\right|^{2}+2 \kappa^{n}\left|h_{a, c}^{n}\right|^{2}}$. Eqn. (3) can be simplified as

$$
\gamma_{a \rightarrow b}^{n}=\frac{P_{c}^{n}\left|h^{n}\right|^{2}}{\sigma^{2}+P_{p}^{n}\left|g^{n}\right|^{2}},
$$

The channel capacity of $a \rightarrow b$ link using $n$-th subcarrier, i.e., $\Sigma_{a \rightarrow b}^{n}$, can be written as

$$
\Sigma_{a \rightarrow b}^{n}=\frac{1}{3} \log _{2}\left(1+\gamma_{a \rightarrow b}^{n}\right) .
$$

\section{INTERFERENCE TEMPERATURE MODEL}

In this section, we discuss the dynamic spectrum techniques for both $P U s$ and $S U s$. PUs uses various subset of frequency channels according to their access technology. $S U s$ do not have their own licensed frequency channels, but these are authorized to access the frequency channels of $P U s$ on a non-interference basis. Interference Temperature model is a spectrum access technique proposed by FCC of USA in 2003 [4],[5]. This model allows interference up to a preexisting interference floor and pro- 


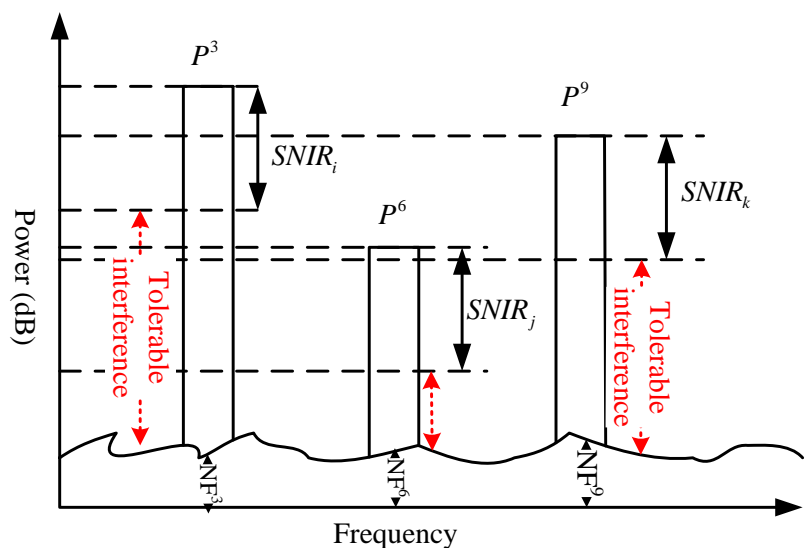

Fig. 3. An interference temperature model which shows the tolerable interference temperature to enable additional communication without the degrading ongoing communication.

vides opportunity to increase over all capacity of the system. $S U s$ have to keep the average interference perceived by a $P U$ receiver below some value. One possible limitation of Interference Temperature model is that it can only regulate average or maximum interference but it can not regulate absolute interference. Let us consider SU nodes $a$ and $b$ are communicating with each other via secondary relay node $c$ and they are sharing frequency bands or carriers with the PU nodes $A, B$ and $C$. The transmission in $n$-th carrier is admissible if

$$
\gamma_{a \rightarrow b}^{n}>\left.\gamma_{a \rightarrow b}^{n}\right|_{\text {threshold }},
$$

or equivalently

$$
Q_{\text {max }}^{n}<P_{\text {max }}^{n}-\left.\gamma_{a \rightarrow b}^{n}\right|_{\text {threshold }}-N F^{n}
$$

where $P_{\max }^{n}$ is the maximum power of the $n$-th subcarrier, $Q_{\max }^{n}$ is the peak interference temperature level, $\left.\gamma_{a \rightarrow b}^{n}\right|_{\text {threshold }}$ is the SNIR threshold, and $N F^{n}$ is the noise figure at the $n$-th subcarrier. This is the allowable interference in carrier $n$ which is illustrated in Fig. 3 Thus the tolerable co-channel interference is $Q_{\max }^{n}<P_{\max }^{n}-\left.\gamma_{a \rightarrow b}^{n}\right|_{\text {threshold }}-N F_{n}$. If we have $M$ pairs of interfere then tolerable co-channel interference is

$$
\frac{Q_{\max }^{n}}{M}<\frac{\left(P_{\max }^{n}-\left.\gamma_{a \rightarrow b}^{n}\right|_{\text {threshold }}-N F^{n}\right)}{M} .
$$

\section{POWER ALLOCATION ALGORITHM}

\subsection{One Primary and One Secondary Links}

The sum capacity maximization problem for $S U s$ can be written as

$$
\max \sum_{n=0}^{N} \frac{B}{3 N} \log _{2}\left(1+\frac{P_{c}^{n}\left|h^{n}\right|^{2}}{\sigma^{2}+P_{p}^{n}\left|g^{n}\right|^{2}}\right) .
$$

The constraints are

$$
\begin{aligned}
\sum_{n=0}^{N} P_{c}^{n} & \leq P_{\max }, \\
P_{c}^{n} f^{n 2} & \leq Q_{\max }^{n}, \\
\frac{1}{N} \sum_{n=0}^{N} P_{c}^{n} f^{n 2} & \leq Q_{\text {ave }}
\end{aligned}
$$

where

$$
\left|f^{n}\right|^{2}=\max \left[\kappa^{n}\left(\left|f_{a B}^{n}\right|^{2},\left|f_{a C}^{n}\right|^{2}\right),\left(1-\kappa^{n}\right)\left(\left|f_{c A}^{n}\right|^{2},\left|f_{c B}^{n}\right|^{2}\right)\right] \text {. }
$$

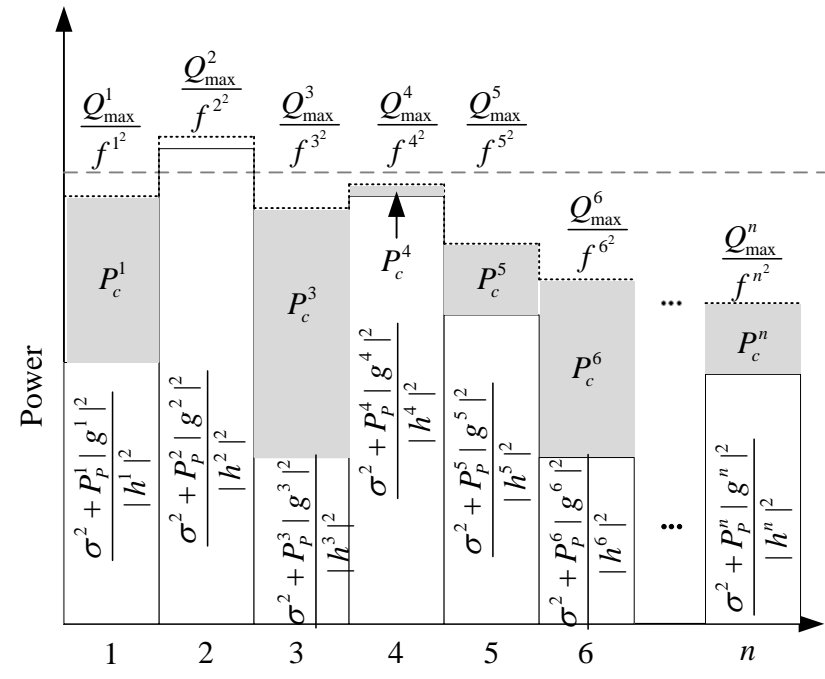

Fig. 4. Multi-level water filling algorithm for the power allocation on different sub-carriers based on the interference temperature limit.

$Q_{\max }^{n}$ and $Q_{a v e}$ are the peak and average interference temperature levels respectively. Eqn. (8) ensures the sum of the allocated power over subcarriers is less than $P_{\max }$; Eqn. (9) is the maximum amount of interference temperature at $n$-th subcarrier is less than $Q_{\max }^{n}$, where $Q_{\max }^{n}=P_{\max }^{n} /\left(k N B_{c}\right)$ and $P_{\max }^{n}$ is the maximum allocated transmit power over $n$-th subcarrier; Eqn. (10) is the average interference level over all subcarriers which is less than $Q_{\text {ave }}$.

4.1.1 Total-transmit and Peak-interference-powers. In this case, the sum capacity maximization problem is optimized subject to the total transmit-power, given in Eqn. (8), and peak interference-power, given in Eqn. (9). The average interference level over all subcarriers is relaxed. The solution of this optimization problem can be written as [9].

$$
P_{c}^{n}=\left[\frac{\Xi}{\lambda}-\frac{\sigma^{2}+P_{p}^{n}\left|g^{n}\right|^{2}}{\left|h^{n}\right|^{2}}\right]^{\frac{Q_{\max }^{n}}{f^{n 2}}},
$$

where $\Xi=\frac{B}{3 N \ln 2}, \lambda$ is the non-negative Lagrange multiplier. It is chosen such that

$$
\sum_{n=0}^{N} P_{c}^{n}(\lambda)=\min \left[P_{\max }, \sum_{n=0}^{N} \frac{Q_{\max }^{n}}{\left|f^{n}\right|^{2}}\right] .
$$

4.1.2 Total-transmit and Average-interference-powers. In this case, the sum capacity maximization problem is optimized subject to the total transmit-power, given in Eqn. (8), and average interference-power, given in Eqn. (10). The peak interference power condition is relaxed. The solution of this optimization problem can be written as 9 .

$$
P_{c}^{i}=\left[\frac{W}{\lambda+\eta\left|f^{n}\right|^{2}}-\frac{\sigma^{2}+P_{p}^{n}\left|g^{n}\right|^{2}}{\left|h^{n}\right|^{2}}\right]^{+} .
$$

where $\lambda$ and $\eta$ are non-negative Lagrange multipliers. Fig. 4 shows power allocation over different subcarriers. Multi-level water filling algorithm is employed for the power allocation on different subcarriers based on the interference temperature limit. 


\subsection{One Primary and $M$-Secondary Links}

The sum capacity maximization problem for the multiple $S U$ can be formulated as

$\max \sum_{n=0}^{N} \sum_{m=0}^{M} \frac{B}{3 N} \log _{2}\left(1+\frac{P_{c_{m}}^{n}\left|h_{m}^{n}\right|^{2}}{\sigma^{2}+P_{p}^{n}\left|g^{n}\right|^{2}+\sum_{j=0}^{M} P_{c_{j}}^{n}\left|h_{j}^{n}\right|^{2}}\right)$

where $j \neq m, M$ is the total number of active $S U$ links, $P_{c_{m}}^{n}$ and $h_{m}^{n}$ are the power and link gain of the $m$-th $S U$ using $n$-th subcarrier respectively.

The constraints are

$$
\begin{array}{r}
\sum_{m=0}^{M} \sum_{n=0}^{N} P_{c_{m}}^{n} \leq P_{\text {max }}, \\
\sum_{m=0}^{M} P_{c_{m}}^{n} f_{m}^{n 2} \leq Q_{\text {max }}^{n}, \\
\sum_{m=0}^{M} \frac{1}{N} \sum_{n=0}^{N} P_{c_{m}}^{n} f_{m}^{n 2} \leq Q_{a v e}
\end{array}
$$

where $f_{m}^{n}$ is the maximum equivalent channel gain of the interference link from $m$-th SU to PU using $n$-th subcarrier.

For the total-transmit and peak-interference-powers, the solution of the optimization problem can be written as [9], [10] .

$$
P_{c_{m}}^{n}=\left[\frac{\Xi}{\lambda}-\frac{\sigma^{2}+P_{p}^{n}\left|g^{n}\right|^{2}+\sum_{j=0}^{M} P_{c_{j}}^{n}\left|h_{j}^{n}\right|^{2}}{\left|h_{m}^{n}\right|^{2}}\right]^{\frac{Q_{\max }^{n}}{f_{m}^{n} 2}}
$$

where $\Xi=\frac{B}{3 N \ln 2}, \lambda$ is the non-negative Lagrange multiplier. It is chosen such that

$$
\sum_{n=0}^{N} P_{c_{m}}^{n}(\lambda)=\min \left[P_{\max }, \sum_{n=0}^{N} \frac{Q_{\max }^{n}}{\left|f_{m}^{n}\right|^{2}}\right]
$$

Spectral efficiency, denoted by $\eta_{n, \gamma}$ can be defined by the following metric

$$
\eta_{N, \gamma}=\frac{N}{v_{N, \gamma}},
$$

where $v_{N, \gamma}$ is the number of channel as a function of $N$ and $\gamma$. Eqn. 20 evaluates the number of communication pairs that can be supported by each channel. The higher the value of $\eta_{N, \gamma}$, the better the spectrum efficiency.

\section{SIMULATION AND RESULTS}

This section includes the simulation results for the proposed NC$\mathrm{CCN}$ by means of Monte-Carlo simulations. We consider all the wireless nodes use OFDM based transmission, the channel fading model is 3-Rayleigh-multipath, the number of subcarriers, i.e., $N=16, Q_{\max }^{i}=Q_{\max }$ and the distribution of noise power over all the subcarrier is same. The simulation is run for 5000 times. Optimal (OPT) power allocation algorithm follows multilevel water-filling whereas sub-optimal (SUB) power allocation follows even power allocation. The power allocation of primary network can be either OPT or SUB whereas the power allocation in a NCCCN can also be either OPT or SUB.Thus the spectral efficiency of a $S U$ with respect to $P U$ depends on OPT/OPT; SUB/OPT; OPT/SUB and SUB/SUB. The simulation parameters for both NCCCN and CCN without NC are considered same for the fair comparison.

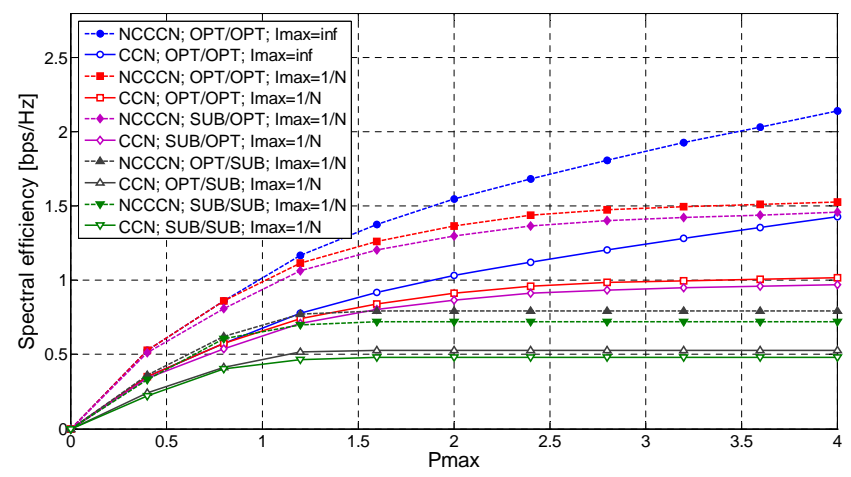

Fig. 5. Effect of $P_{\max }$ on spectral efficiency considering $I_{\max }$.

Fig. 5 shows the effect of $P_{\max }$ on spectral efficiency for different constraint of $I_{\max }$. When no maximum-interference constraint is imposed, i. e., $I_{\max }=\infty$, the spectral efficiency of $S U$ is highest and follows logarithmic trend. On the other hand, when the maximum-interference temperature constraint is considered, i.e., $I_{\max }=1 / N$, the spectral efficiency of $S U$ follows the logarithmic trend for smaller value of $P_{\max }$ but becomes flat for higher value of $P_{\max }$ for all OPT/OPT; SUB/OPT; OPT/SUB and SUB/SUB cases. The maximum-interference temperature constraint imposes limit on maximum power allocation to avoid interference to the primary network. The dash-sign curves represent the spectral efficiency of the proposed network whereas the solid-sign curves represents the spectral efficiency of CCN without ANC. The spectral efficiency [bit-per-second/Hz] of the proposed NCCCN is better than that of CCN without ANC [9]. The ANC requires less radio resources compared to traditional CCN to finish a bi-directional communication. It is also found that the spectral efficiency of OPT/OPT is better than that of SUB/OPT; OPT/SUB; SUB/SUB for the interference limited case.

Fig. 6 shows the effect of $P_{\max }$ on spectral efficiency for different constraint of $I_{\text {ave }}$. When no average-interference constraint is imposed, i. e., $I_{\text {ave }}=\infty$, the spectral efficiency of $S U$ is highest and follows logarithmic trend. On the other hand, when the average-interference temperature constraint is considered, i.e., $I_{\text {ave }}=1 / N$, the spectral efficiency of $S U$ follows the logarithmic trend for smaller value of $P_{\max }$ but becomes flat for higher value of $P_{\max }$ for all OPT/OPT; SUB/OPT; OPT/SUB and SUB/SUB cases. The average-interference temperature constraint imposes limit on power allocation to avoid interference to the primary network. It is also found that the spectral efficiency of OPT/OPT is better than that of SUB/OPT; OPT/SUB; SUB/SUB for the interference limited case. The proposed NC$\mathrm{CCN}$ also performs better than that of CCN without ANC [9] in this case.

Figs. 7 and 8 show the effect of $I_{\max }$ on spectral efficiency considering $P_{\max }$ and $P_{\text {ave }}$ constraints respectively. In terms of spectral efficiency, the proposed NCCCN outperforms the CCN considered in [9].

The relay position also effect the spectral efficiency as shown in Fig. 9. In all cases the spectral efficiency is the maximum if relay is placed midway between source and destination. It proves the Lemma in [9]

Fig. 10 shows the sum capacity as a function of number of SUs. The sum capacity initially increases with the number of SUs. When the number of active SU link is less than 4, The sum capacity increases with SU links as shown in Fig. 10. On the other hand, when the number of active SU link is higher than 8 , the interference from SU links to PU link increases. Thus the sum capacity/user decreases.

Fig. 11 shows the effect of SNIR threshold on the spectral efficiency. Here, the spectrum efficiency is plotted as a function 


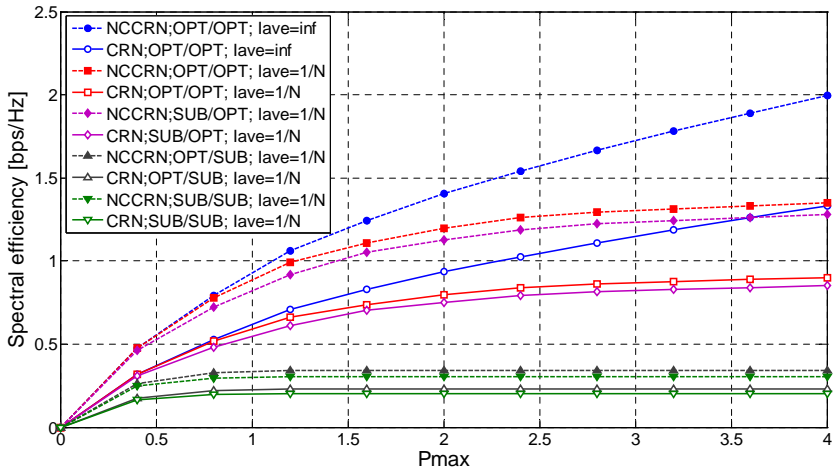

Fig. 6. Effect of $P_{\max }$ on spectral efficiency considering $I_{a v e}$.

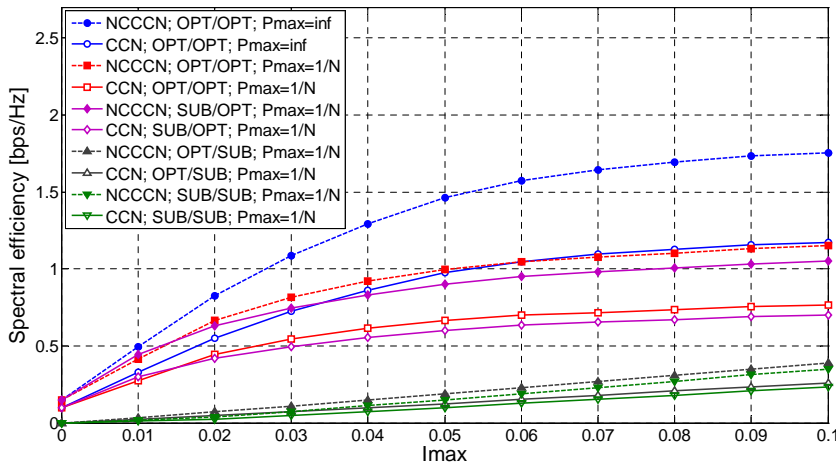

Fig. 7. Effect of $I_{\max }$ on spectral efficiency considering $P_{\max }$.

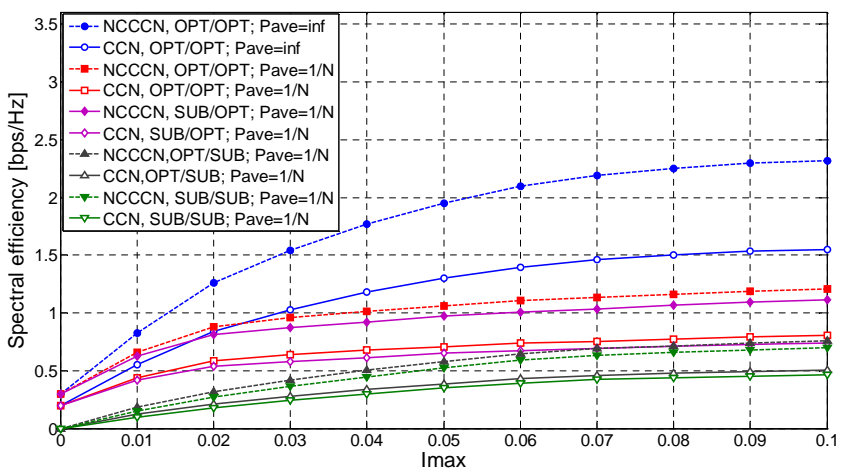

Fig. 8. Effect of $I_{\max }$ on spectral efficiency considering $P_{a v e}$.

of $M$ and SNIR threshold. When the number of communication pairs increase, i.e., network becomes more congested, spectral efficiency decreases for the same value of SNIR. It means that spectrum requirement increases if the network becomes more congested. But in all the cases spectral efficiency decreases with the increase in SNIR threshold which proves the Eqn. 20.

\section{CONCLUSION}

In this paper, we proposed the network coded-cognitive cooperative network (NCCCN). The power allocation optimization problem is formed under peak and average interference constraints considering interference temperature constraints. The proposed system performs better than the single hop cognitive radio network. Simulation results shows that the optimal power allocation achieves higher data rate than suboptimal power allocation. The

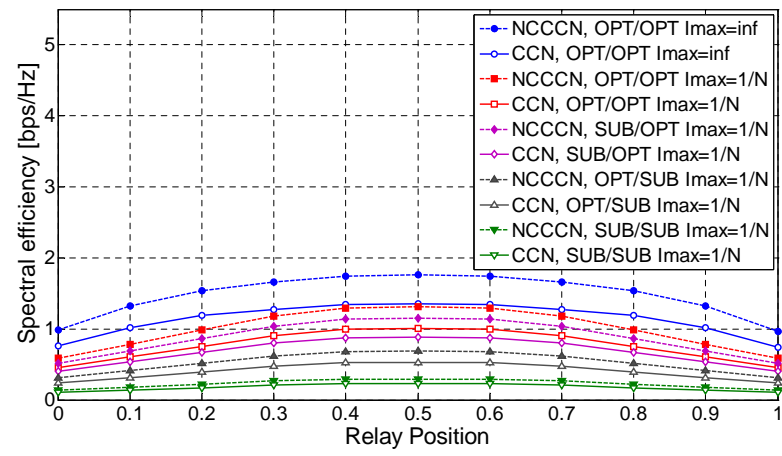

Fig. 9. Effect of relay position on the spectral efficiency

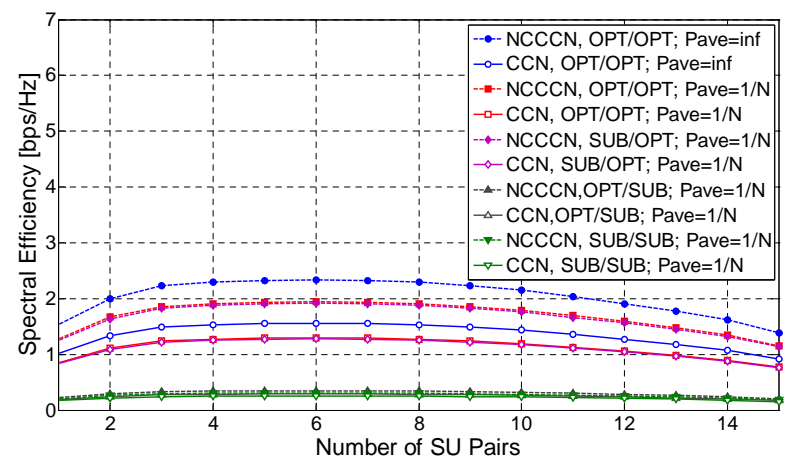

Fig. 10. Effect of number of active SUs on the SUs capacity.

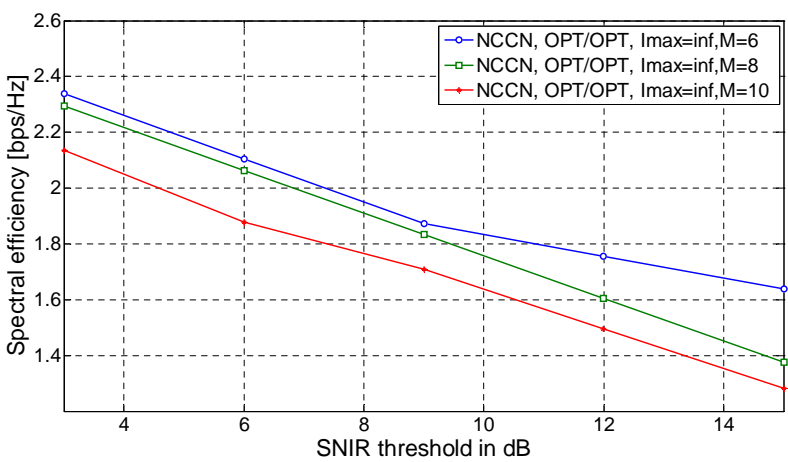

Fig. 11. Effect of SNIR threshold on the spectral efficiency.

performance of the system decreases as the number of SU increases. We can extend our work considering bit allocation and Optimal power allocation based on outage probability analysis.

\section{Acknowledgement}

The early version of this work has been presented orally in WiCOM 2011, China and WCNIS 2010, China. We would like to thank all antonymous reviews for their suggestions.

\section{REFERENCES}

[1] Sunita S. Barve and P. Kulkarni. A performance based routing classification in cognitive radio networks. International Journal of Computer Applications, 44(19), 2012. 
[2] Y. Chen, S. Kishore, and J. Li. Wireless diversity through network coding. In IEEE WCNC, USA, 2006. IEEE Press.

[3] S. Valentin D. H. Woldegebreal and H. Karl. Outage probability analysis of cooperative transmission protocols without and with network coding: inter-user channels based comparison. In MSWiM '07: Proceedings of the 10th ACM Symposium on Modeling, analysis, and simulation of wireless and mobile systems, USA, 2007. ACM.

[4] FCC. Establishment of interference temperature metric to quantify and manage interference and to expand available unlicensed operation in certain fixed mobile and satellite frequency bands. ET Docket 03-222, Notice of Inquiry and Proposed Rulemaking, Dec 2003.

[5] FCC. Establishment of interference temperature metric to quantify and manage interference and to expand available unlicensed operation in certain fixed mobile and satellite frequency bands. ET Docket 03-289, Notice of Inquiry and Proposed Rulemaking, Nov 2003.

[6] FCC Spectrum Policy Task Force. Fcc report of the spectrum efficiency working group, Nov. 2002.

[7] H.Gacanin and F.Adachi. Broadband analog network coding. IEEE Transations on Wireless Communications, 9(5), May 2010.

[8] M. S. Kaiser and K. M. Ahmed. Performance analysis of network coded bidirectional relaying in ofdm networks. In ICECE 2010, USA, 2010. IEEE Press.

[9] M. S. Kaiser, K. M. Ahmed, and R. A. Shah. Power allocation in ofdm-based cognitive relay networks. In IEEE In- ternational Conference on Wireless Communications, Networking and Information Security (WCNIS), pages 202206, USA, 2010. IEEE.

[10] M. Shamim Kaiser. Power allocation for the network coded cognitive cooperative network. In IEEE International Conference on Wireless Communications, Networking and Mobile Computing (WiCOM), pages 1-5, USA, 2011. IEEE.

[11] S. Katti, I. Maric, A. Goldsmith, D. Katabi, and M. Mdard. Joint relaying and network coding in wireless networks. In IEEE international Symposium on Information Theory, USA, 2007. IEEE Press.

[12] J. Mitola. An integrated agent architechture for sofeware define radio. Ph.D. Thesis, KTH Royel Institute of Technology, Sweden, 2000.

[13] S. F. Shirazi, S. H. Shirazi, S. M. Shah, and M. K. Shahid. Hybrid spectrum sensing algorithm for cognitive radio network. International Journal of Computer Applications, 45(17), 2012.

[14] K. Son, B. C. Jung, and D. K. Sung. Opportunistic underlay transmission in multi-carrier cognitive radio systems. IEEE WCNC, 2009.

[15] D. H. Woldegebreal and H. Karl. Network-Coding-Based Cooperative Transmission in Wireless Sensor Networks: Diversity-Multiplexing Tradeoff and Coverage Area Extension, chapter Lecture Notes in Computer Science, pages 83-99. Springer Berlin - Heidelberg, 2008. 